\title{
Mechanized Verification of Computing Dominators for Formalizing Compilers
}

\author{
Jianzhou Zhao and Steve Zdancewic \\ University of Pennsylvania \\ \{jianzhou, stevez\}@cis.upenn.edu
}

\begin{abstract}
One prerequisite to the formal verification of modern compilers is to formalize computing dominators, which enable SSA forms, advanced optimizations, and analysis. This paper provides an abstract specification of dominance analysis that is sufficient for formalizing modern compilers; it describes a certified implementation and instance of the specification that is simple to design and reason about, and also reasonably efficient. The paper also presents applications of dominance analysis: an SSA-form type checker, verifying SSA-based optimizations, and constructing dominator trees. This development is a part of the Vellvm project. All proofs and implementation have been carried out in Coq.
\end{abstract}

\section{Introduction}

Compilers are not always correct due to the complexity of language semantics and transformation algorithms, the trade-offs between compilation speed and verifiability, etc. Bugs in compilers can undermine the source-level verification efforts (such as type systems, static analysis, and formal proofs), and produce target programs with different meaning from source programs. The CompCert project [12] first implemented a realistic and mechanically verified compiler with classic intermediate representations in the Coq proof assistant. The CompCert compiler generates compact and efficient assembly code for a large fragment of the $\mathrm{C}$ language, and is proved to be more robust than non-verified compilers.

Recently researchers started to formalize and verify modern compilers in the Vellvm project [14 and in the CompCertSSA project [3. One crucial component of modern compilers, such as LLVM and GCC, is computing dominators - on a control-follow-graph, a node $l_{1}$ dominates a node $l_{2}$ if all paths from the entry to $l_{2}$ must go through $l_{1}[2$. Dominance analysis allows compilers to represent programs in the SSA form [6] (which enables many advanced SSA-based optimizations), optimize loops, analyze memory dependency, and parallelize code automatically, etc. Therefore, one prerequisite to the formal verification of modern compilers is to formalize computing dominators.

In this paper, we present the formalization of dominance analysis used in the Vellvm project. To the best of our knowledge, this is the first mechanized verification of dominator computation for LLVM. Although the CompCertSSA project [3] also formalized dominance analysis to prove the correctness of a global value numbering optimization, our results are more general: beyond soundness, we establish completeness and related metatheory results that can be used in 
other applications. Because different styles of formalization may also affect the cost of proof engineering, we also discuss some tradeoffs in the choices of formalization. In this work, we evaluate our formalism by applying it to several applications in Vellvm.

To simplify the formal development, we describe the work in the context of Vminus, which is a simpler subset of the full LLVM SSA IR formalized in Vellvm, that still captures the essence of dominance analysis. Our Coq development formalizes all the claims of the paper for the full Vellvm ${ }^{1}$. Following LLVM, we distinguish dominators at the block level and at the instruction level. Given the former one, we can easily compute the latter one. Therefore, we will focus on the block-level analysis, and discuss the instruction-level analysis only briefly.

We present the following contributions. Section 2 gives a specification of computing dominators at the block level. We instantiate the specification by two algorithms. Section 3 shows the standard dominance analysis [1 (AC). Section 4 presents an extension of $\mathrm{AC}$ [5] (CHK) that is easy to implement and verify, but still fast. We verify the correctness of both algorithms. Section 3.1 provides a verified depth first search algorithm. Then, Section 5 extends the dominance analysis to the instruction level, and present several applications used in the Vellvm project: a type checker for SSA, verifying SSA-based optimizations, and constructing dominator trees. Section 6 evaluates performance of the algorithms, and shows that in practice CHK runs nearly as fast as the LLVM's algorithm.

\section{The Specification of Computing Dominators}

This section first defines dominators in term of the syntax of Vminus, then gives an abstract and succinct specification of algorithms that compute dominators.

Syntax of Vminus. Figure 1 gives the syntax of Vminus, focusing on the syntax of Vminus at the block level. Section 5 will revisit the rest of the syntax. All code in Vminus resides in top-level functions, whose bodies are composed of blocks $b$. Here, $\bar{b}$ denotes a list of blocks; we use similar notation for other lists throughout the paper. As in classic compiler representations, a basic block consists of a label $l$, a series of instructions insn followed by a terminator tmn (br and ret) that branches to another block or returns from the function. In the following, we also use the label of a block to denote the block.

The set of blocks making up the top-level function $f$ constitutes a controlflow graph (CFG) $G=(e$, succs) where $e$ is the entry point (the first block) of $f$; succs maps each label to a list of its successors. On a CFG, we use $G=l_{1} \rightarrow^{*} l_{2}$ to denote a path $\rho$ from $l_{1}$ to $l_{2}$, and $l \in \rho$ to denote that $l$ is in the path $\rho$. By wf $f$, we require that a well-formed function must contain an entry point that cannot be reached from other blocks, all terminators can only branch to blocks within $f$, and that all labels in $f$ are unique. In this paper, we consider only well-formed functions to streamline the presentation.

\footnotetext{
${ }_{1}^{1}$ Available at http://www.cis.upenn.edu/ jianzhou/Vellvm/dominance
} 


\begin{tabular}{|c|c|c|}
\hline Types & typ $::=$ int & Instructions insn $::=\phi|c|$ tmn \\
\hline Constants & cnst $::=$ Int & Phi Nodes $\quad \phi::=r=$ phi $\operatorname{typ}{\overline{\left[v a l_{j}, l_{j}\right]}}^{j}$ \\
\hline Values & val $::=r \mid$ cnst & Commands $\quad c::=r:=v_{a l}$ bop $_{\text {val }}$ \\
\hline Blocks & $b::=l \bar{\phi} \bar{c} t m n$ & Terminators tmn $::=\mathbf{b r} l \mid \mathbf{b r}$ val $l_{1} l_{2} \mid$ ret typ val \\
\hline Functions & $f::=\operatorname{fun}\{\bar{b}\}$ & $\hat{\phi}::=c \mid t m n$ \\
\hline
\end{tabular}

Fig. 1. Syntax of Vminus.

Definition 1 (Domination (Block-level)). Given $G$ with an entry e,

- A block $l$ is reachable, written $G \rightarrow^{*} l$, if there exists a path $G \models e \rightarrow^{*} l$.

- A block $l_{1}$ dominates a block $l_{2}$, written $G \models l_{1} \gg=l_{2}$, if for every path $\rho$ from $e$ to $l_{2}, l_{1} \in \rho$.

- A block $l_{1}$ strictly dominates a block $l_{2}$, written $G=l_{1} \gg l_{2}$, if for every path $\rho$ from $e$ to $l_{2}, l_{1} \neq l_{2} \wedge l_{1} \in \rho$.

Because the dominance relations of a function at the block level and in its CFG are equivalent, in the following we do not distinguish $f$ and $G$. The following consequence of the definitions are useful to define the specification of computing dominators. For all labels in $G, \gg=$ and $\gg$ are transitive.

\section{Lemma 1.}

- If $G \models l_{1} \gg=l_{2}$ and $G \models l_{2} \gg=l_{3}$, then $G \models l_{1} \gg=l_{3}$.

- If $G \models l_{1} \gg l_{2}$ and $G \models l_{2} \gg l_{3}$, then $G \models l_{1} \gg l_{3}$.

However, because there is no path from the entry to unreachable labels, $\gg=$ and $\gg$ relate every label to any unreachable labels.

Lemma 2. If $\neg\left(G \rightarrow^{*} l_{2}\right)$, then $G \models l_{1} \gg=l_{2}$ and $G \models l_{1} \gg l_{2}$.

If we only consider the reachable labels in $V, \gg$ is acyclic.

Lemma 3 (» is acyclic). If $G \rightarrow^{*} l$, then $\neg G \models l \gg l$.

Moreover, all labels that strictly dominate a reachable label are ordered.

Lemma 4 (》 is ordered). If $G \rightarrow^{*} l_{3}, l_{1} \neq l_{2}, G \models l_{1} \gg l_{3}$ and $G \models l_{2} \gg l_{3}$, then $G \models l_{1} \gg l_{2} \vee G \mid=l_{2} \gg l_{1}$.

\subsection{Specification}

Coq Notations. We use \{\} to denote an empty set; use $\{+\},\{<=\}$, ' $\operatorname{In}^{\text {' }},\{\backslash /\}$ and $\{\bigwedge\}$ to denote set addition, inclusion, membership, union and intersection respectively. Our developments reuse the basic tree and map data structures implemented in the CompCert project [12: ATree.t and PTree.t are trees with keys of type $l$ and positive respectively; PMap.t is a map with keys of type positive. We use [] to denote tree and map lookup. succs are defined by trees. [] returns an empty list when a searched-for key in succs does not exist. [x] is a list with one element $\mathrm{x}$. 


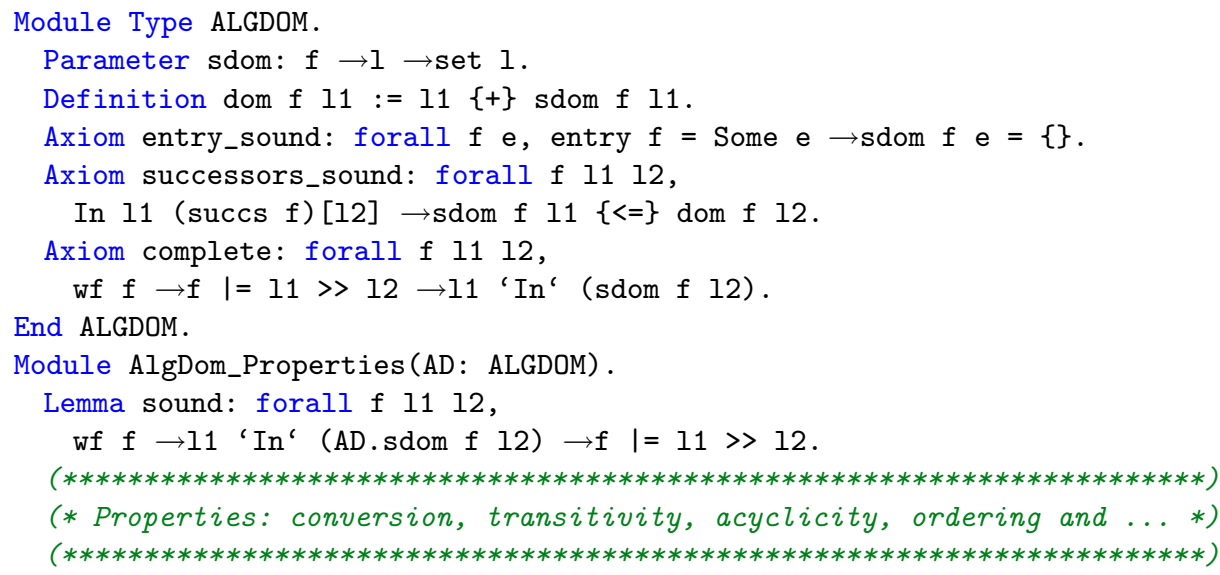

Fig. 2. The specification of algorithms that find dominators.

Figure 2 gives an abstract specification of algorithms that compute dominators using a Coq module interface ALGDOM. First of all, sdom defines the signature of a dominance analysis algorithm: given a function $f$ and a label $l_{1}$, (sdom $\left.f l_{1}\right)$ returns the set of strict dominators of $l_{1}$ in $f$; dom defines the set of dominators of $l_{1}$ by adding $l_{1}$ into $l_{1}$ 's strict dominators.

To make the interface simple, ALGDOM only requires the basic properties that ensure that sdom is correct: it must be both sound and complete in terms of the declarative definitions (Definition 1). Given the correctness of sdom, the AlgDom_Properties module can 'lift' properties (conversion, transitivity, acyclicity, ordering, etc.) from the declarative definitions to the implementations of sdom and dom. Section 5 shows how clients of ALGDOM use the properties proven in AlgDom_Properties by examples.

ALGDOM requires completeness directly. Soundness can be proven by two more basic properties: entry_sound requires that the entry has no strict dominators; successors_sound requires that if $l_{1}$ is a successor of $l_{2}$, then $l_{2}$ 's dominators must include $l_{1}$ 's strict dominators. Given an algorithm that establishes the two properties, AlgDom_Properties proves that the algorithm is sound by induction over any path from the entry to $l_{2}$.

\subsection{Instantiations}

In the literature, there is a long history of algorithms that find dominators, each making different trade-offs between efficiency and simplicity. Most of the industry compilers, such as LLVM and GCC, use the classic Lengauer-Tarjan algorithm [1] (LT) that has a complexity of $O(E * \log (N))$ where $N$ and $E$ are the number of nodes and edges respectively, but is complicated to implement and reason about. The Allen-Cocke algorithm [1] (AC) based on iteration is easier to design, but suffers from a large asymptotic complexity. Moreover, LT 


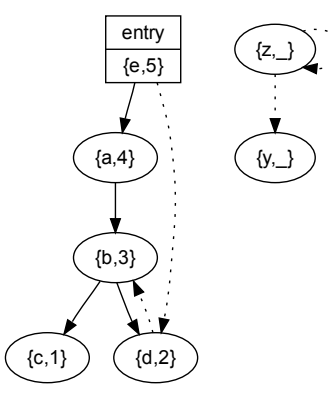

Fig. 3. Postorder.

\begin{tabular}{|c|c|c|}
\hline stk & visited & PO_12p po \\
\hline $\mathrm{e}[\mathrm{a} \mathrm{d}]$ & $\mathrm{e}$ & \\
\hline $\mathrm{e}[\mathrm{d}] ; \mathrm{a}[\mathrm{b}]$ & e a & \\
\hline $\mathrm{e}[\mathrm{d}] ; \mathrm{a}[] ; \mathrm{b}[\mathrm{cd}]$ & e a b & \\
\hline $\mathrm{e}[\mathrm{d}] ; \mathrm{a}[] ; \mathrm{b}[\mathrm{d}] ; \mathrm{c}[]$ & e a b c & $(\mathrm{c}, 1)$ \\
\hline $\mathrm{e}[\mathrm{d}] ; \mathrm{a}[] ; \mathrm{b}[] ; \mathrm{d}[\mathrm{b}]$ & e a b c d & $(\mathrm{c}, 1)$ \\
\hline $\mathrm{e}[\mathrm{d}] ; \mathrm{a}[] ; \mathrm{b}[] ; \mathrm{d}[]$ & e a b c d & $(\mathrm{c}, 1) ;(\mathrm{d}, 2)$ \\
\hline $\mathrm{e}[\mathrm{d}] ; \mathrm{a}[] ; \mathrm{b}[] ;$ & $\mathrm{e} a \mathrm{bcd}$ & $(\mathrm{c}, 1) ;(\mathrm{d}, 2) ;(\mathrm{b}, 3)$ \\
\hline $\mathrm{e}[\mathrm{d}] ; \mathrm{a}[] ;$ & e a b c d & $(\mathrm{c}, 1) ;(\mathrm{d}, 2) ;(\mathrm{b}, 3) ;(\mathrm{a}, 4)$ \\
\hline$e[]$ & e a b c d & $(\mathrm{c}, 1) ;(\mathrm{d}, 2) ;(\mathrm{b}, 3) ;(\mathrm{a}, 4) ;(\mathrm{e}, 5)$ \\
\hline
\end{tabular}

Fig. 4. The DFS execution sequence.

explictly creates dominator trees that provide convenient data structures for compilers whereas AC needs an additional tree construction algorithm with more overhead. The Cooper-Harvey-Kennedy algorithm [5] (CHK), extended from AC with careful engineering, runs nearly as fast as LT in common cases [5], but is still simple to implement and reason about. Moreover, CHK generates dominator trees implicitly, and provides a faster tree construction algorithm.

Because CHK gives a relatively good trade-off between verifiability and efficency, we present CHK as an instance of ALGDOM. In the following sections, we first review the AC algorithm, and then study its extension CHK.

\section{The Allen-Cocke Algorithm}

The Allen-Cocke algorithm (AC) is an instance of the forward worklist-based Kildall's algorithm [10] that visits nodes in reverse postorder (PO) 9] (in which $\mathrm{AC}$ converges faster). At the high-level, our Coq implementation of AC works in three steps: 1) calculate the PO of a CFG by depth-first-search (DFS); 2) compute strict dominators for PO-numbered nodes in Kildall; 3) finally relate the analysis results to the original nodes. We omit the 3rd step's proofs here.

This section first presents a verified DFS algorithm that computes PO, then reviews Kildall's algorithm as implemented in the CompCert project [12, and finally it studies the implementation and metatheory of AC.

\subsection{DFS: PO-numbering}

DFS starts at the entry, visits nodes as deep as possible along each path, and backtracks when all deep nodes are visited. DFS generates PO by numbering a node after all its children are numbered. Figure 3 gives a PO-numbered CFG. In the CFG, we represent the depth-first-search (DFS) tree edges by solid arrows, and non-tree edges by dotted arrows. We draw the entry node in a box, and other nodes in circles. Each node is labeled by a pair with its original label name on the left, and its PO number on the right. Because DFS only visits reachable nodes, the PO numbers of unreachable nodes are represented by '.'.

Figure 5 shows the data structures and auxiliary functions used by a typical DFS algorithm that maintains four components to compute PO. PostOrder 


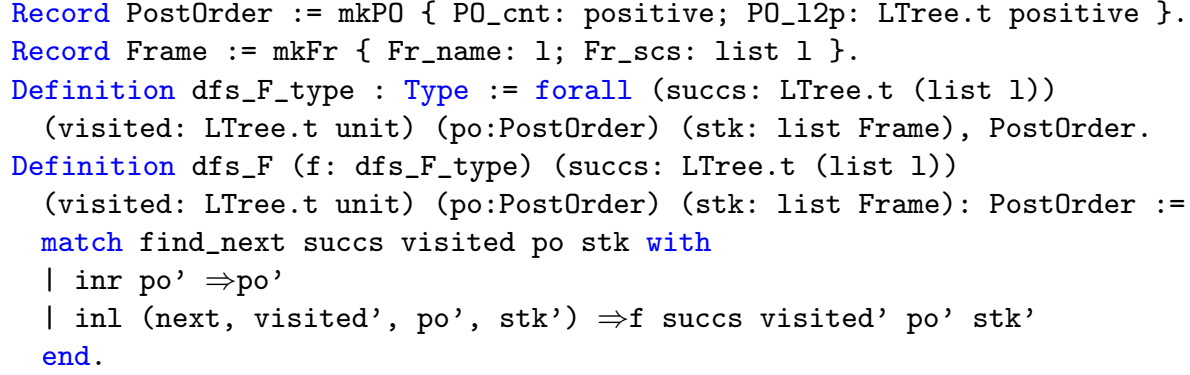

Fig. 5. The DFS algorithm.

takes the next available PO number and a map from nodes to their PO numbers with type positive. succs maps a node to its successors. To facilitate reasoning about DFS, we represent the recursive information of DFS explicitly by a list of Frame records that each contains a node Fr_name and its unprocessed successors Fr_scs. To prevent the search from revisiting nodes, the DFS algorithm uses visited to record visited nodes. df $\mathbf{s}_{-} F$ defines one recursive step of DFS.

Figure 4 gives a DFS execution sequence (by running dfs_F until all nodes are visited) of the CFG in Figure 3. We use $l\left[l_{1} \cdots l_{n}\right]$ to denote a frame with the node $l$ and its unprocessed successors $l_{1}$ to $l_{n} ;(l, p)$ to denote a node $l$ and its PO $p$. Initially the DFS adds the entry and its successors to the stack. At each recursive step, find_next finds the next available node that is the unvisited node in the Fr_scs of the latest node $l^{\prime}$ of the stack. If the next available node exists, the DFS pushes the node with its successors to the stack, and makes the node to be visited. find_next pops all nodes in front of $l^{\prime}$, and gives them PO numbers. If find_next fails to find available nodes, the DFS stops.

We can see that the straightforward algorithm is not a structural recursion. To implement the algorithm in Coq, we must show that it terminates. Although in Coq we can implement the algorithm by well-founded recursion, such designs are hard to reason about 4. One possible alternative is implementing DFS with a 'strong' dependent type to specify the properties that we need to reason about DFS. However, this design is not modular because when the type of DFS is not strong enough - for example, if we need a new lemma about DFS - we must extend or redesign its implementation by adding new invariants. Instead, following the ideas in Coq'Art 4, we implement DFS by iteration and prove its termination and inductive principle separately. By separating implementation and specification, the DFS design is modular, and easier to reason about.

Figure 6 presents our design. The top-level entry is iter, which needs a bounding step $\mathrm{n}$, a fixpoint $\mathrm{F}$ and a default value $\mathrm{g}$. iter only calls $\mathrm{g}$ when $\mathrm{n}$ reaches zero, and otherwise recursively calls one more iteration of $\mathrm{F}$. If $\mathrm{F}$ is terminating, we can prove that there must exist a final value and a bound $\mathrm{n}$, such that for any bound $\mathrm{k}$ that is greater than or equal to $\mathrm{n}$, iter always stops and generates the same final value. In other words, $\mathrm{F}$ reaches a fixpoint in fewer 


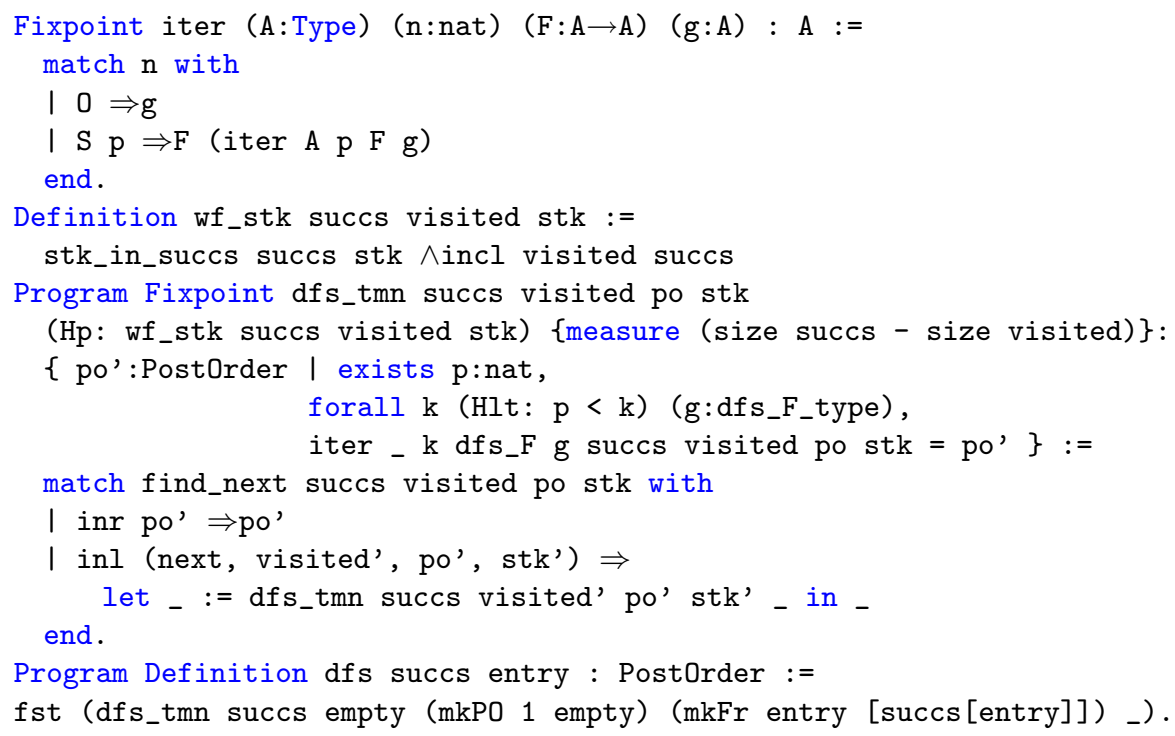

Fig. 6. Termination of the DFS algorithm.

than $\mathrm{n}$ steps. The proof of the existence of $\mathrm{n}$ is erasable; the computation part provides a terminating algorithm, not requiring the bound step at runtime.

In Figure 6, dfs_tmn proves DFS termination, which is established by wellfounded recursion over the number of unvisited nodes. This holds because each iteration the DFS visits more nodes. The invariant that the number of unvisited nodes decreases holds only for well-formed recursion states (wf_stk), which requires that all visited nodes and unprocessed nodes in frames are in the CFG.

To reason about dfs, we defined a well-founded inductive principle for $\mathrm{df} s$ (See our code). With the inductive principle, we proved the following properties of DFS that are useful to establish the correctness of AC and CHK.

Variable (succs: ATree.t (list 1)) (entry:1) (po:PostOrder). Hypothesis Hdfs: dfs succs entry = po.

First of all, a non-entry node must have at least one predecessor that has a greater PO number than the node's. This is because 1) DFS must visit at least one predecessor of a node before visiting the node; 2) PO gives greater numbers to the nodes visited earlier:

Lemma dfs_order: forall $11 \mathrm{p} 1,11<$ entry $\rightarrow($ PO_12p po $)[11]=$ Some p1, exists 12 , exists $\mathrm{p} 2$,

In 12 ((make_preds succs) [11] $) \wedge($ P0_12p po) [12] = Some p2 $\wedge$ p2 $>$ p1.

(* Given succs, (make_preds succs) computes predecessors of each node. *)

Second, a node is PO-numbered iff the node is reachable:

Lemma dfs_reachable:forall 1, (PO_12p po) [1] $<>$ None $\longleftrightarrow$ (entry, succs) $\rightarrow * 1$. Moreover, different nodes do not have the same PO number.

Lemma dfs_inj: forall $1112 \mathrm{p}$,

$\left(\mathrm{PO} \_12 \mathrm{p}\right.$ po $)[12]=$ Some $\mathrm{p} \rightarrow\left(\mathrm{PO} \_12 \mathrm{p}\right.$ po $)[11]=$ Some $\mathrm{p} \rightarrow 11=12$. 


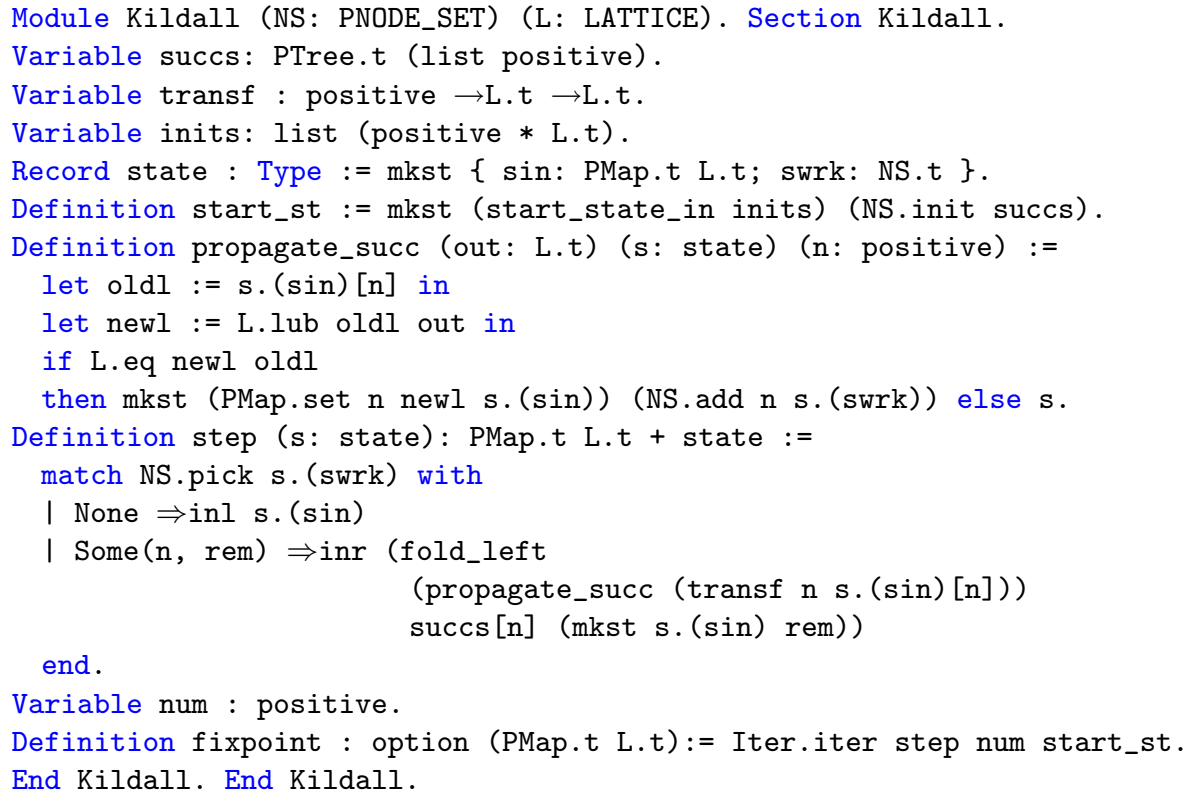

Fig. 7. Kildall's algorithm.

\subsection{Kildall's algorithm}

Figure 7 summarizes the Kildall module used in the CompCert project. The module is parameterized by the following components: NS that provides the order to process nodes, and a lattice L that defines top, bot, equality (eq), least upper bound (lub) and order (ge) of the abstract domain of an analysis; succs that is a tree that maps each node to its successors; transf that is the transfer function of Kildall analysis; inits that initializes the analysis. Given the inputs, state records the iteration states that include sin, which records analysis states for each node, and a work list swrk containing nodes to process.

The fixpoint implements iterations by Iter.iter - bounded recursion with a maximal step number (num) 4]. Iter.iter is partial if an analysis does not stop after the maximal number of steps. A monotone analysis must reach its fixpoint after a finite number of steps. Therefore, we can alway pick a large enough number of steps for a monotone analysis.

Initially Kildall's algorithm calls start_st to initialize iteration states. Nodes not in inits are initialized to be the bottom of L. Then start_st adds all nodes into the worklist and starts loops. step defines the loop body. At step, Kildall's algorithm checks if there are still unprocessed nodes in the worklist. If the worklist is empty, the algorithm stops. Otherwise, step picks a node from the worklist in term of the order provided by NS, and then propagates its information (computed by transf) to all the node's successors by propagate_succ. In propagate_succ, the new value of a successor is L.lub of its old value and the 
propagated value from its predecessor. The algorithm only adds a successor into the worklist when its value is changed.

Kildall's algorithm satisfies the following properties:

Variable res: PMap.t L.t.

Hypothesis Hfix: fixpoint = Some res.

First of all, the worklist contains nodes that have unstable successors in the current state. Formally, each state st preserves the following invariant:

forall $\mathrm{n}$, NS.In $\mathrm{n}$ st. (swrk) $V$

(forall s, In s (succs[n]) $\rightarrow$ L.ge st. (sin)[s] (transf n st. (sin)[n])).

Each iteration may only remove the picked node $\mathrm{n}$ from the worklist. If none of n's successors' values are changed, no matter whether $\mathrm{n}$ belongs to its successors, $\mathrm{n}$ won't be added back to the worklist. Therefore, the above invariant holds. This invariant implies that when the analysis stops, all nodes hold the in-equations:

Lemma fixpoint_solution: forall s,

In $\mathrm{s}$ ( $\operatorname{succs}[\mathrm{n}]$ ) $\rightarrow$ L.ge res[s] (transf $\mathrm{n}$ res[n]).

The second property of Kildall's algorithm is monotonicity. At each iteration, the value of a successor of the picked node can only be updated from oldl to newl. Because newl is the least upper bound of oldl and out, newl is greater than or equal to oldl. Therefore, iteration states are always monotonic:

Lemma fixpoint_mono: incr (start_state_in inits) res.

where incr is a pointwise lift of L.ge for corresponding nodes. With monotonicity, we proved that Kildall's algorithm must terminate (See our code).

\subsection{The AC Algorithm}

AC instantiates Kildall with PN that picks nodes in reverse PO (by picking the maximal nodes from the worklist), and LDoms that defines the lattice of AC. Dominance analysis computes a set of strict dominators for each node. We represent the domain of LDoms by option (set 1). The top and bot of LDoms are Some nil and None respectively. The least upper bound, order and equality of LDoms are lifted from set intersection, set inclusion, and set equality to option: None is smaller than Some $\mathrm{x}$ for any $\mathrm{x}$. This design leads to better performance by providing shortcuts for operations on None. Note that using None as bot does not make the height of LDoms to be infinite, because any non-bot element can only contain nodes in the CFG, and the height of LDoms is $N$.

$\mathrm{AC}$ uses the following transfer function and initialization:

Definition transf 11 input := $11\{+\}$ input.

Definition inits $:=[(e$, LDoms.top $)]$.

Initially AC sets the strict dominators of the entry to be empty, and other nodes' strict dominators to be all labels in the function. The algorithm will iteratively remove non-strict-dominators from the sets until the conditions below hold (by Lemma fixpoint_mono and Lemma fixpoint_solution):

(forall s, In $\mathrm{s}$ (succs $[\mathrm{n}]$ ) $\rightarrow$

$$
\text { L.ge (st. }(\sin ))[\mathrm{s}](\mathrm{n}\{+\}(\mathrm{st} .(\sin ))[\mathrm{n}])) \wedge(\mathrm{st} .(\sin ))[\mathrm{e}]=\{\} \text {. }
$$

which proves that AC satisfies entry_sound and successors_sound. 
To show that the algorithm is complete, it is sufficient to show that each iteration state st preserves the following invariant:

forall $\mathrm{n} 1 \mathrm{n} 2, \sim \mathrm{n} 1$ 'In' st. (sin) [n2] $\rightarrow \sim(\mathrm{e}$, succs) $\mid=\mathrm{n} 1>\mathrm{n} 2$.

In other words, $\mathrm{AC}$ only removes non-strict dominators. Initially, $\mathrm{AC}$ sets the entry's strict dominators to be empty. Because in a well-formed CFG, the entry has no predecessors, the invariant holds at the very beginning. At each iteration, suppose that we pick a node $\mathrm{n}$, and updates one of its successors $\mathrm{s}$. Consider a node $\mathrm{n}$ ' not in LDoms.lub st. (sin) [s] ( $\{+\}$ st. (sin) [n]). If $n$ ' is not in LDoms.lub st. ( $\sin )[s]$, then $n$ ' does not strictly dominate $s$ because st holds the invariant. If $\mathrm{n}^{\prime}$ is not in ( $\mathrm{n}\{+\}$ st. ( $\left.\left.\sin \right)[\mathrm{n}]\right)$, then $\mathrm{n}^{\prime}$ does not strictly dominate $\mathrm{n}$ because st holds the invariant. Appending the path from the entry to $\mathrm{n}$ that bypasses $\mathrm{n}$ ' with the edge from $\mathrm{n}$ to $\mathrm{s}$ leads to a path from the entry to $\mathrm{s}$ that bypasses $\mathrm{n}^{\prime}$. Therefore, $\mathrm{n}$ ' does not strictly dominate $\mathrm{s}$, either.

\section{Extension: the Cooper-Harvey-Kennedy Algorithm}

The CHK algorithm is based on the following observation: when AC processes nodes in a reversed post-order (PO), if we represent the set of strict dominators in a list, and always add a newly discovered strict dominator at the head of the list (on the left in Figure 9), the list must be sorted by PO. Figure 9 shows the execution of the algorithm for the CFG in Figure 3.

Because lists of strict dominators are always sorted, we can implement the set intersection (lub) and the set comparison (eq) of two sorted lists by traversing the two lists only once. Moreover, the algorithm only calls eq after lub. Therefore, we can group lub and eq into LDoms. lub together. The following defines a merge function used by LDoms.lub that intersects two sorted lists and returns whether the final result is equal to the left one:

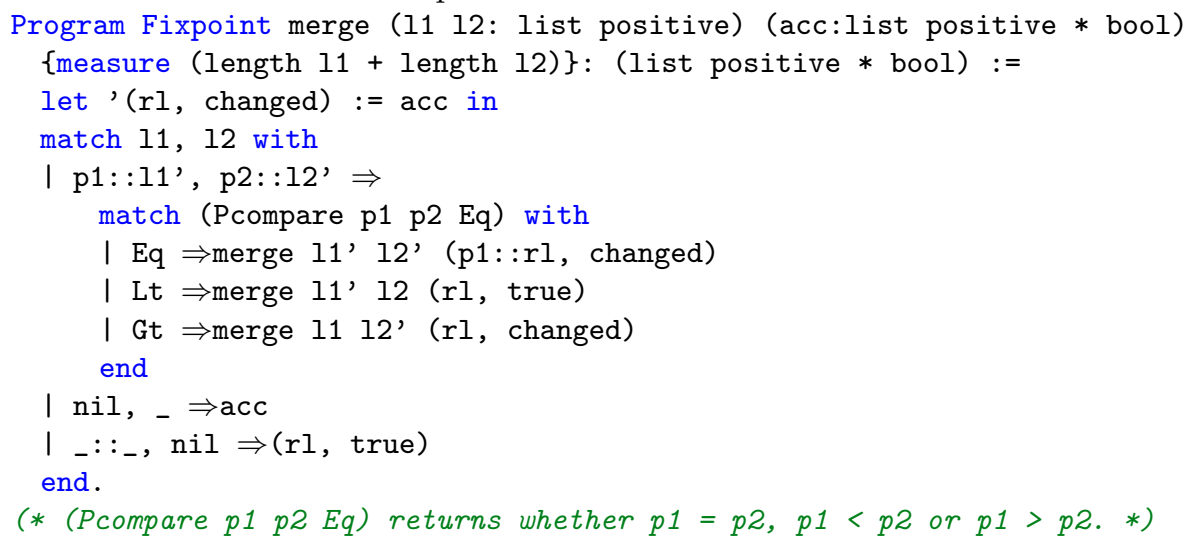

\subsection{Correctness}

To show that CHK is still correct, it is sufficient to show that all lists are wellsorted at each iteration, which ensures that the above merge correctly implements intersection and comparison. First, if a node with number $\mathrm{n}$ still maps to bot, the worklist must contain one of its predecessors that has a greater number. 


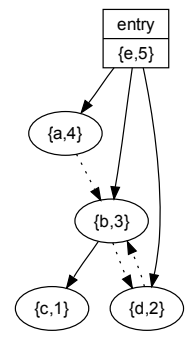

\begin{tabular}{|l||l|l|l|l|l|l|l|l|l|}
\hline Nodes & \multicolumn{10}{|c|}{ sin } \\
\hline 5 & {[]} & {[]} & {[]} & {[]} & {[]} & {[]} & {[]} & {[]} & {[]} \\
4 & $\cdot$ & {$[5]$} & {$[5]$} & {$[5]$} & {$[5]$} & {$[5]$} & {$[5]$} & {$[5]$} & {$[5]$} \\
3 & $\cdot$ & $\cdot$ & {$[45]$} & {$[45]$} & {$[45]$} & {$[5]$} & {$[5]$} & {$[5]$} & {$[5]$} \\
2 & $\cdot$ & $\cdot$ & $\cdot$ & {$[345]$} & {$[345]$} & {$[345]$} & {$[35]$} & {$[35]$} & {$[35]$} \\
1 & $\cdot$ & {$[5]$} & {$[5]$} & {$[5]$} & {$[5]$} & {$[5]$} & {$[5]$} & {$[5]$} & {$[5]$} \\
\hline swrk & {$[54321][[4321]$} & {$[321][[21]$} & {$[1]$} & {$[3]$} & {$[21]$} & {$[1]$} & {[]} \\
\hline
\end{tabular}

Fig. 8. Dominator Trees.

Fig. 9. The execution of CHK.

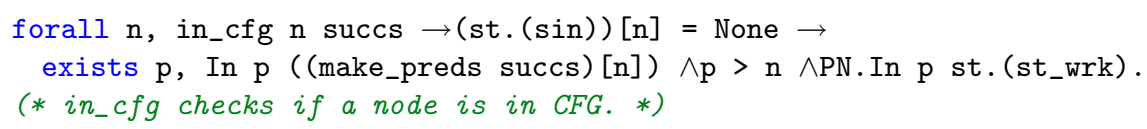

This invariant holds in the beginning because all nodes are in the worklist. At each iteration, the invariant implies that the picked node $\mathrm{n}$ with the maximal number in st. (st_wrk) is not bot. Suppose it is bot, there cannot be any node with greater number in the worklist. This property ensures that after each iteration, the successors of $\mathrm{n}$ cannot be bot, and that the new nodes added into the worklist cannot be bot, because they must be those successors. Therefore, the predecessors of the remaining bot nodes still in the worklist cannot be $\mathrm{n}$. Since only $\mathrm{n}$ is removed, the rest of the bot nodes still hold the above invariant.

In the algorithm, a node's value is changed from bot to non-bot when one of its non-bot predecessors is processed. With the above invariant, we know that the predecessor must be of larger number. Once a node turns to be non-bot, no new elements will be added in its set. Therefore, this implies that, at each iteration, if the value of a node is not bot, then all its candidate strict dominators must be larger than the node:

forall $\mathrm{n}$ sdms, (st. ( $\sin )$ ) [n] = Some sdms $\rightarrow$ Forall (Plt n) sdms.

(* Plt is the less-than of positive. *)

Moreover, a node $\mathrm{n}$ is considered as a candidate of strict dominators originally by tranf that always cons $\mathrm{n}$ at the head of (st. (sin)) [n]. Therefore, we proved that the non-bot value of a node is always sorted:

forall $\mathrm{n}$ sdms, (st. (sin)) [n] = Some sdms $\rightarrow$ Sorted Plt (n::sdms).

\section{$5 \quad$ Applications}

\section{$5.1 \quad$ Type Checker}

The first application is the type checker of Vminus. The Vminus language in Figure 1 is in SSA form 6 in which each variable may be defined only once, statically, and each use of the variable must be dominated by its definition with respect to the control-flow graph of the containing function. To maintain these invariants in the presence of branches and loops, SSA form uses $\phi$-instructions to merge definitions from different incoming paths. As usual in SSA representation, 
the $\phi$ nodes join together values from a list of predecessor blocks of the controlflow graph - each $\phi$ node takes a list of (value, label) pairs that indicates the value chosen when control transfers from a predecessor block with the associated label.

To check that a program is in SSA form, we need to extend domination relations from the block-level to the instruction-level. Instruction positions are denoted by program counters $p c$. We write $f[p c]=\lfloor$ insn $\rfloor$ if insn is at $p c$ of $f$.

\section{Definition 2 (Domination (Instruction-level)).}

$-v a l$ uses $r \triangleq v a l=r$.

- insn uses $r \triangleq \exists$ val.val uses $r \wedge$ val is an operand of insn.

- A variable $r$ is defined at a program counter pc of $f$, written $f$ defines $r @ p c$, if $f[p c]=\lfloor$ insn $\rfloor$ and $r$ is the left-hand side of insn.

- In function $f, p c_{1}$ strictly dominates $p c_{2}$, written $f \models p c_{1} \gg p c_{2}$, if $p c_{1}$ and $p c_{2}$ are at distinct blocks $l_{1}$ and $l_{2}$ respectively and $f \models l_{1} \gg l_{2}$; or if $p c_{1}$ and $p c_{2}$ are in the same block, and $p c_{1}$ appears earlier than $p c_{2}$.

- $\mathbf{s d o m}_{f}(p c)$ is the set of variables whose definitions strictly dominate $p c$ : $\left\{r \mid f\right.$ defines $r @ p c^{\prime}$ and $\left.f \mid=p c^{\prime} \gg p c\right\}$

Then we check if a program is of SSA form with the following rules:

$$
\frac{\forall r .\left(\hat{\phi} \mathbf{u s e s} r \Longrightarrow r \in \operatorname{sdom}_{f}(p c)\right)}{f \digamma_{\succ} \hat{\phi} @ p c} \quad \frac{\overline{\forall r_{j} \cdot\left(v a l_{j} \mathbf{u s e s}_{j} \Longrightarrow r_{j} \in \mathbf{s d o m}_{f}\left(l_{j} \cdot \mathbf{t}\right)\right)}}{f \digamma_{\succ} r=\operatorname{phi} t y p{\overline{\left[v a l_{j}, l_{j}\right]}}^{j}}
$$

The left rule ensures that a non- $\phi$-instruction ( $c$ or $t m n)$ can only use the definitions in the scope of $\mathbf{s d o m}_{f}(p c)$; the right rule ensures that in $\phi$, an incoming value must use the definition that strictly dominates the end of the corresponding incoming block where $l$. $t$ is the program counter at the end of $l$. Please refer to 14 for the type safety proofs of Vminus.

\subsection{SSA-based Optimizations}

The SSA form is good for implementing optimizations because the SSA invariants make def/use information of variables explicit, enforcing fewer mutable states [2. An SSA-based transformation is correct if it preserves the semantics of the original program and its transformed program is still in SSA. Here, we briefly show how to reason about well-formedness-preservation by examples.

First, we proved that the strict domination relation at the instruction level still satisfies transitivity and acyclicity.

\section{Lemma 5.}

- If $f=p c_{1} \gg p c_{2}$ and $f=p c_{2} \gg p c_{3}$, then $f \mid=p c_{1} \gg p c_{3}$.

- If pc is in a reachable block, then $\neg f \models p c \gg p c$.

Consider the following typical SSA-based optimization: 


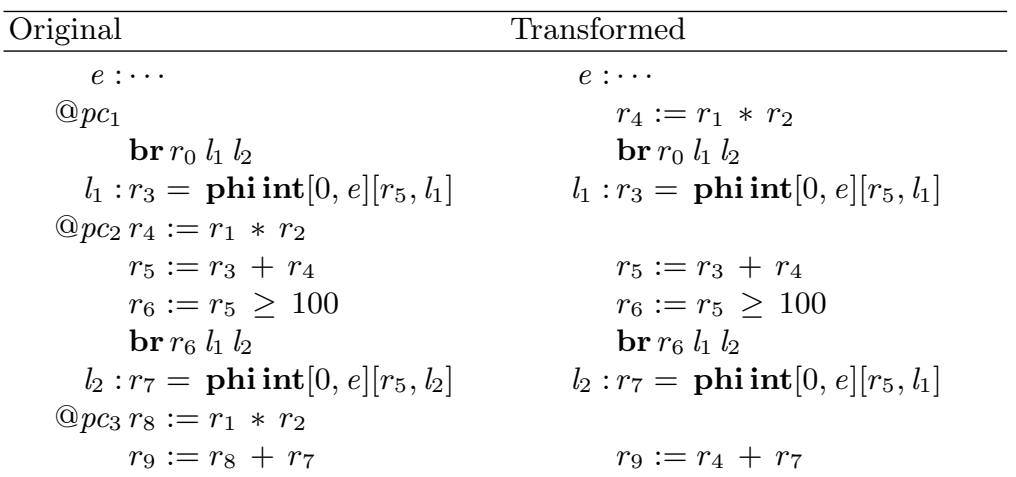

In the original program, $r_{1} * r_{2}$ is a partial common expression for the definitions of $r_{4}$ and $r_{8}$, because there is no domination relation between $r_{4}$ and $r_{8}$. Therefore, eliminating the common expression directly is not correct.

We might transform this program in three steps. First, we move the instruction $r_{4}:=r_{1} * r_{2}$ from $l_{1}$ to the end of $e$. Because $e$ strictly dominates $l_{1}$, we have $f=p c_{1} \gg p c_{2}$ where $p c_{1}$ is exactly before $e . \mathbf{t} ; f$ defines $r_{4} @ p c_{2}$. By Lemma 5, the definition of $r_{4}$ at $p c_{1}$ should still strictly dominate all its uses.

We have $f=p c_{1} \gg p c_{3}$ where $f$ defines $r_{8} @ p c_{3}$, because $e$ strictly dominates $l_{2}$. Then, we can safely replace all the uses of $r_{8}$ by $r_{4}$, because the definition of $r_{4}$ at $p c_{1}$ dominates all the uses of $r_{8}$ (by Lemma 5).

Finally, by Lemma 5 , we know that $r_{4}$ and $r_{8}$ cannot be equal. Therefore, we can remove $r_{8}$, because there are no uses of $r_{8}$ after the substitution. The final program after the transformations is shown on the right of the above example.

\subsection{Constructing Dominator Trees}

In practice, compilers construct dominator trees from dominators, and analyze or optimize programs by recursion on dominator trees.

\section{Definition 3.}

- A block $l_{1}$ is an immediate dominator of a block $l_{2}$, written $G=l_{1} \ggg l_{2}$, if $G=l_{1} \gg l_{2}$ and $\left(\forall G \models l_{3} \gg l_{2}, G \mid=l_{3} \gg=l_{1}\right)$.

- A tree is called a dominator tree of $G$ if the tree has an edge from $l$ to $l^{\prime}$ iff $G \models l \ggg l^{\prime}$.

Figure 8 shows the dominator tree of a CFG, in which solid edges represent tree edges, and dotted edges represent non-tree but CFG edges. Formally, a dominator tree has the inductive well-formed property with which we can reason about recursion on dominator trees: given a tree node $l, 1) l$ is reachable; 2) $l$ is different from all labels in $l$ 's descendants; 3) labels of $l$ 's subtrees are disjointed; 4) $l$ immediate-dominates its children; 5) $l$ 's subtrees are well-formed.

Consider the final analysis results of CHK in Figure 9, we can see that for each node, its list of strict dominators exactly presents a path from root to the node on the dominator tree. Therefore, we can construct a dominator tree by 


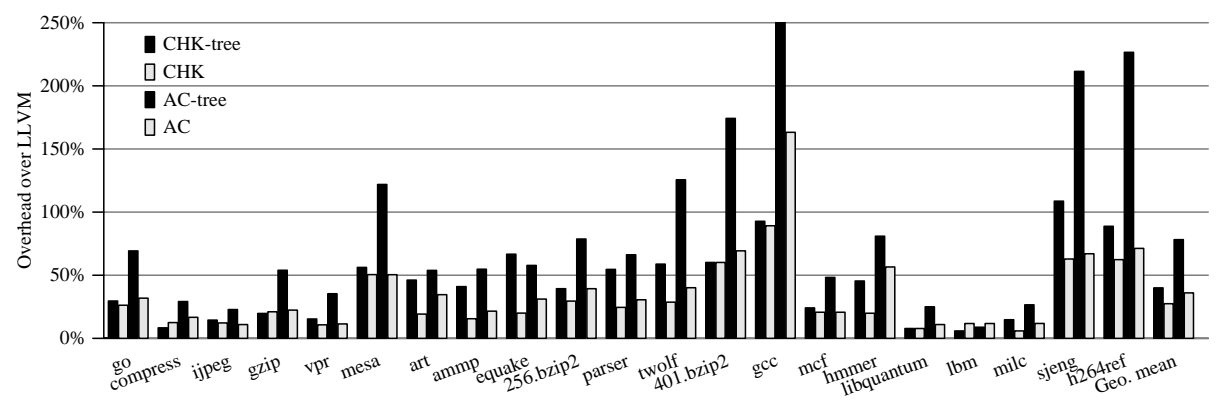

Fig. 10. Analysis overhead over LLVM's dominance analysis for our extracted analysis.

merging the paths. We proved that the algorithm correctly constructs a wellformed dominator tree (See our code). For the sake of space, we only present that each tree edge represents $\ggg$ by showing that for any node $l$ in the final state, the list of l's dominators must be sorted by $\ggg$.

We first show that the list is sorted by $\gg$. Consider two adjacent nodes in the list, $l_{1}$ and $l_{2}$, such that $l_{1}<l_{2}$. Because of soundness, $G \models l_{1} \gg=l$ and $G \models l_{2} \gg=l$. By Lemma $4, G=l_{2} \gg l_{1} \vee G \models l_{1} \gg l_{2}$. Suppose $G \models l_{1} \gg l_{2}$, by completeness, $l_{1}$ must be in the strict dominators computed for $l_{2}$, and therefore, be greater than $l_{2}$. This is a contradiction. Then, we prove that the list is sorted by $\gg$. Suppose $G=l_{3} \gg l_{1}$. By Lemma $11 G l_{3} \gg l$. By completeness, $l_{3}$ must be in the list. We have two cases: 1) $l_{3} \geq l_{2}$ : because the list is sorted by $\left.\gg, G \models l_{3} \gg=l_{2} ; 2\right) l_{3} \leq l_{1}$ : this is a contradiction by Lemma 3

\section{Performance Evaluation}

We use Coq extraction to obtain a certified implementation of AC and CHK. We evaluate the performance of the resultant code on a $1.73 \mathrm{GHz}$ Intel Core i7 processor with $8 \mathrm{~GB}$ memory running benchmarks selected from the SPEC CPU benchmark suite that consist of over $873 \mathrm{k}$ lines of $\mathrm{C}$ source code.

Figure 10 reports the analysis time overhead (smaller is better) over the LLVM dominance analysis (which uses LT) baseline. LT only generates dominator trees. Given a dominator tree, the strict dominators of a tree node are all the node's ancestors. The second left bar of each group shows the overhead of CHK, which provides an average overhead of $27.45 \%$. The right-most bar of each group is the overhead of AC, which provides $36.02 \%$ on average.

To study the asymptotic complexity, Figure 11 shows the result of graphs that elicit the worst-case behavior used in 8 . On average, CHK is 86.59 times slower than LT. The ',' indicates that the running time is too long to collect. For the testcases on which AC stops, AC is 226.14 times slower than LT.

The results of CHK match earlier experiments 815): in common cases, CHK runs nearly as fast as LT. For programs with reducible CFGs, a forward iteration analysis in reverse PO halts in no more than 6 passes [9, and most CFGs of the benchmarks are reducible. The worst-case tests contain huge irreducible CFGs. 


\begin{tabular}{|l|l|l|l|l|l|l|l|}
\hline \multicolumn{3}{|c|}{ Instance } & \multicolumn{5}{c|}{ Analysis Times (s) } \\
\hline Name & Vertices & Edges & LT & CHK & CHK-tree & AC & AC-tree \\
\hline idfsquad & 6002 & 10000 & 0.08 & 10.54 & 24.87 & - & - \\
ibfsquad & 4001 & 6001 & 0.14 & 11.38 & 13.16 & 12.43 & 30.00 \\
itworst & 2553 & 5095 & 0.14 & 8.47 & 11.22 & 19.16 & 69.72 \\
sncaworst & 3998 & 3096 & 0.19 & 17.03 & 32.08 & 205.07 & 740.53 \\
\hline
\end{tabular}

Fig. 11. Worst-case behavior.

Different from these experiments, AC does not provide large overhead, because we use None to represent bot, which provides shortcuts for set operations.

As shown in Section 5.3. CHK computes dominator trees implicitly, while AC needs additional costs to create dominator trees. Figure 10 and Figure 11 also report the performance of the dominator tree construction. CHK-tree stands for the algorithm that first computes dominators by CHK, and then runs the tree construction defined in Section 5.3. AC-tree stands for the algorithm that first computes dominators by AC, sorts strict dominators for each node, and then runs the same tree construction. For common programs, on average, CHK-tree provides an overhead $40.00 \%$ over the baseline; AC-tree provides an overhead $78.20 \%$ over the baseline (gcc's overhead is $361.23 \%$ ). The additional overhead of AC-tree is from its sorting algorithm. For worst-case programs, on average, CHK-tree is 104.48 times slower than LT. For the testcases on which AC-tree stops, on average, AC-tree is 738.24 times slower than LT.

These results indicate that CHK makes a good trade-off between simplicity and efficiency.

\section{Related Work}

Machine-checked formalizations. The Vellvm project 14 uses dominance analysis to design a type checker of LLVM bitcode in SSA form. This paper extends and generalizes the implementation and metatheory in the Vellvm project. The CompCertSSA project [3] improves the CompCert compiler by creating a verified SSA-based middle-end. They also formalize the AC algorithm to validate SSA construction and GVN passes, and prove the soundness of AC. We implement both $\mathrm{AC}$ and $\mathrm{CHK}$ - an extension of $\mathrm{AC}$ in a generic way, and prove they are both sound and complete. We also provide the corresponding dominator tree constructions, and evaluate performance.

Informal formalizations. Georgiadis and Tarjan [7] propose an almost lineartime algorithm that validates if a tree is a dominator tree of a CFG. Although the algorithm is fast, it is nearly as complicated as the LT algorithm, and it requires a substantial amount of graph theory. Ramalingam 13 proposes another dominator tree validation algorithm by reducing validating dominator trees to validating loop structures. However, in practice, most of modern loop identification algorithms used in LLVM and GCC are based on dominance analysis to find loop headers and bodies. 


\section{Conclusion}

This paper provided an abstract specification of dominance analysis that is crucial for compiler design/verification and program analysis. We implemented and certified an instance of the specification that has a good trade-off between efficiency and simplicity. We also presented several applications of the analysis: a type checker for the SSA form; verifying SSA-based optimizations; and constructing dominator trees. This development is a part of the Vellvm project. However, our work might be used in other compiler verification projects [3].

Acknowledgments We thank Santosh Nagarakatte and Milo Martin whose valuable discussions and technical input helped us carry out this research. This research was sponsored in part by NSF grant CCF-1065116. The views and conclusions contained in this document are those of the authors and should not be interpreted as representing the official policies, either expressed or implied, of the U.S. Government.

\section{References}

1. F. E. Allen and J. Cocke. Graph theoretic constructs for program control flow analysis. Technical report, IBM T.J. Watson Research Center, 1972.

2. A. W. Appel. Modern Compiler Implementation in C: Basic Techniques. Cambridge University Press, 1997.

3. G. Barthe, D. Demange, and D. Pichardie. A formally verified SSA-based middleend - Static Single Assignment meets CompCert. In ESOP '12, 2012.

4. Y. Bertot and P. Castéran. Interactive Theorem Proving and Program Development. Coq'Art: The Calculus of Inductive Constructions, 2004.

5. K. D. Cooper, T. J. Harvey, and K. Kennedy. A simple, fast dominance algorithm. Available online at www.cs.rice.edu/ keith/Embed/dom.pdf, 2000.

6. R. Cytron, J. Ferrante, B. K. Rosen, M. N. Wegman, and F. K. Zadeck. Efficiently computing static single assignment form and the control dependence graph. ACM Trans. Program. Lang. Syst., 13:451-490, 1991.

7. L. Georgiadis and R. E. Tarjan. Dominator tree verification and vertex-disjoint paths. In SODA '05, pages 433-442, 2005.

8. L. Georgiadis, R. F. Werneck, R. E. Tarjan, and D. I. August. Finding dominators in practice. In ESA '04, pages 677-688, 2004.

9. J. B. Kam and J. D. Ullman. Global data flow analysis and iterative algorithms. J. ACM, 23(1):158-171, Jan. 1976.

10. G. A. Kildall. A unified approach to global program optimization. In $P O P L$ ' 73 , pages 194-206, 1973.

11. T. Lengauer and R. E. Tarjan. A fast algorithm for finding dominators in a flowgraph. ACM Trans. Program. Lang. Syst., 1:121-141, 1979.

12. X. Leroy. A formally verified compiler back-end. Journal of Automated Reasoning, 43(4):363-446, 2009.

13. G. Ramalingam. On loops, dominators, and dominance frontiers. ACM Trans. Program. Lang. Syst., 24(5):455-490, 2002.

14. J. Zhao, S. Nagarakatte, M. M. K. Martin, and S. Zdancewic. Formalizing the LLVM intermediate representation for verified program transformations. In POPL '12, 2012. 